\title{
Insulin receptor substrate 1 overexpression promotes survival of glioblastoma cells through AKT1 activation
}

\author{
Gokhan Gorgisen, Zafer Yaren \\ Department of Medical Biology, Faculty of Medicine, Van Yuzuncu Yil University, Turkey
}

\begin{abstract}
Glioblastoma multiforme (GBM) is the most common and malignant type of central nervous system tumours in adults. Strict regulation of glucose homeostasis has a significant role in GBM pathogenesis. Insulin receptor substrate 1 (IRS1) protein is the most important adaptor molecule involved in the regulation of glucose metabolism. It interacts with many cancer-related receptors and its overexpression is strongly associated with cell proliferation and survival. Our study was aimed to understand the role of IRS1 proteins in GBM cell viability. U-87 MG cells were transfected with PCDNA3.1-flagtagged-human IRS1 expression vector. Insulin induced phosphorylation levels of IRS1, AKT1 and ERK1/2 and Grb2 expression were examined to determine the effects of ectopic IRS1 overexpression on insulin signalling and the viability levels of U-87 MG cells were determined by MTT analysis. Overexpression of IRS1 in U-87 MG cells led to an increase in cell viability. Its overexpression also increased Grb2 expression and phosphorylation of AKT1 through elevation of IRS1 tyrosine phosphorylation in IRS1-transfected U-87 MG cells compared to control and mock transfected groups. Our study showed that increased IRS1 expression and activation may promote the cell viability via AKT1 activation. IRS1 signalling may be considered as a therapeutic target for further studies.
\end{abstract}

Key words: IRS1, GBM, insulin signalling, IGFR.

\section{Introduction}

Glioblastoma multiforme (GBM) is the most common and aggressive primary malignant brain tumour in adults. It constitutes nearly $45 \%$ of all gliomas and has the poorest prognosis. Despite the progress in treatment options such as surgery, chemotherapy and radiotherapy, the survival rate of GBM patients is very low $[16,22]$. Therefore, understanding the molecular pathogenesis of GBM is crucial.

Metabolic complications such as type 2 diabetes increase the risk of cancer development and poorer outcomes [8]. Several studies showed that hypergly- caemia is associated with shorter survival time in GBM patients [1,5]. The recent study has also demonstrated that physiologically relevant insulin concentrations induce cell proliferation in patient derived glioblastoma xenograft models [12]. Therefore, regulation of glucose metabolism has a pivotal role for GBM patients. Insulin signalling is the main cellular pathway that is responsible for glucose homeostasis [6]. Insulin is an anabolic hormone that shows its metabolic and mitogenic effects through insulin and insulin like growth factor receptors (IGFR) [3]. Expression levels of insulin growth factor (IGF) and insulin 
receptors are significantly increased and aberrantly activated in human cancers [23]. Insulin receptor substrate proteins (IRS) are the main adaptor molecule of these receptors. They can also interact with several growth hormone receptors that are strongly correlated with tumour growth [11]. IRS1 is the first member of this family and is widely expressed in human tissues. When IRS1 binds to its receptors, it is phosphorylated on tyrosine residues. Tyrosine phosphorylation of IRS1 induces the activation of PI3K-AKT and Ras-ERK pathways [13]. Transformation capacity of IRS1 was first shown in mouse embryonic fibroblasts and its overexpression is primarily responsible for cell proliferation in breast cancer [4]. In addition to breast cancer, many researchers determined the overexpression and aberrant activation of IRS1 in numerous cancer cell lines and tumour samples and they reported that IRS1 overexpression is associated with lung, prostate, pancreatic, hepatocellular, ovarian cancers and medulloblastoma [18]. To date, the relationship between IRS1 expression and the viability of GBM cells has not been demonstrated.

In view of these findings, this study aimed to determine the effects of IRS1 overexpression on GBM cell viability and insulin signalling pathway.

\section{Material and methods \\ Cell culture and biological reagents}

Monoclonal anti-IRS1, antiphosphotyrosine, antiERK1/2, anti-pERK1/2, anti-AKT1 and pAKT1 antibodies were obtained from Thermo Fisher Scientific (USA); monoclonal b-actin and Grb2 antibodies were obtained from Santa Cruz Biotechnology, Inc. (Santa Cruz, CA); anti-rabbit HRP and anti-mouse HRP were purchased from BioRad (Hercules, CA). U-87 MG cells were grown in DMEM supplemented with $10 \% \mathrm{FBS}$, $100 \mathrm{mg} / \mathrm{ml}$ penicillin, $50 \mathrm{mg} / \mathrm{ml}$ streptomycin, and $1 \mathrm{mM}$ glutamine.

\section{Transfections and treatments}

Sixty to seventy percent confluent U-87 MG cells were transfected with 15 ug of mock or IRS1 expression vectors prepared in flag-tagged pcDNA3.1. Thermo TurboFect Transfection reagent (Thermo Scientific, USA) was used and the reverse transfection method was applied according to the manufacturer's instructions. Overexpression of IRS1 was verified by western blotting using anti-IRS1 antibody. U-87 MG transfected and control cells were cultured in FBS-free medium for $16 \mathrm{~h}$ and treated with $100 \mathrm{ng} / \mathrm{ml}$ insulin for 15 and 30 minutes.

\section{Western blotting}

Control and transfected cells were lysed in Triton X-100 buffer containing 50 mM HEPES, pH 7.0, 150 $\mathrm{mM} \mathrm{NaCl}, 10 \%$ glycerol, $1.2 \%$ Triton X-100, $1.5 \mathrm{mM}$ $\mathrm{MgCl}_{2}, 1 \mathrm{mM}$ EGTA, $10 \mathrm{mM}$ sodium pyrophosphate, $100 \mathrm{mM} \mathrm{NaF}, 1 \mathrm{mM}$ sodium orthovanadate, $1 \mathrm{mM}$ PMSF, 0.15 units $/ \mathrm{ml}$ aprotinin, $10 \mu \mathrm{g} / \mathrm{ml}$ leupeptin, and $10 \mu \mathrm{g} / \mathrm{ml}$ pepstatin A. 75 micrograms of total proteins were separated by SDS-PAGE, then blotted onto PVDF membranes. The membranes were blocked in 1\% BSA in PBST. The blots were first labelled with phospho-specific antibodies for IRS1, AKT, Grb2 and ERK1/2. Labelled blots were then stripped off and re-labelled with antibodies which detect all forms of these proteins. $\beta$-actin was used as a loading control for these blots. Signal intensity on blots was determined using the enhanced chemiluminescent detection system.

\section{MTT assay}

The viability levels of transfected and control U-87 MG cells were detected by the MTT assay. Cells were seeded into the wells of 96-well plates at densities of 5 thousand with 6 replicates in complete DMEM. They were incubated for $24 \mathrm{~h}, 48 \mathrm{~h}$ and $72 \mathrm{~h}$. At the end of incubation, $5 \mathrm{mg} / \mathrm{ml}$ MTT solution was added for 4 hours at $37^{\circ} \mathrm{C}$ in an incubator, then the medium was removed and DMSO was added to dissolve the formazan crystals. The plates were shielded from light using a foil and left in an orbital shaker maintained at 600 revolutions/minute for 5 minutes. The amount of MTT formazan product formed was determined by measuring absorbance (A) at $540 \mathrm{~nm}$, with $690 \mathrm{~nm}$ as the reference wavelength. Statistical analysis was performed using GraphPad Prism version 8.2.0 (San Diego; USA).

\section{Results \\ Effects of IRS1 overexpression on insulin signalling}

To determine the effects of exogenic IRS1 expression on the expression and activation of downstream targets of IRS1, insulin induced IRS1, ERK1/2, AKT1 activation and Grb2 expression level were examined.

First, we detected the changes in insulin induced IRS1 tyrosine phosphorylation in IRS1 overexpressed 
U-87 MG cells. Tyrosine phosphorylation of IRS1 two fold increased after treatment of insulin for $15 \mathrm{~min}$ in control and mock groups compared to untreated matches (Fig. 1). After 30 min insulin treatment, the tyrosine phosphorylation level of IRS1 was 50\% higher compared to untreated groups. Interestingly, untreated IRS1 overexpressed U-87 MG cells showed two-fold increased IRS1 activation compared to control and mock transfected groups. Insulin treatment for $15 \mathrm{~min}$ also led to an increase in the level of IRS1 phosphorylation in the IRS1 overexpressed group compared to its untreated group. Although tyrosine phosphorylation of IRS1 was decreased in control and mock groups after 30 min insulin treatment, we observed an increase in IRS1 tyrosine phosphorylation in IRS1 transfected U-87 MG cells (Fig. 1).

Grb2 is one of the main downstream targets of IRS1 protein. Therefore, we wanted to determine the expression level of Grb2 after insulin treatment in IRS1 overexpressed cells. Based on our western blot analy-

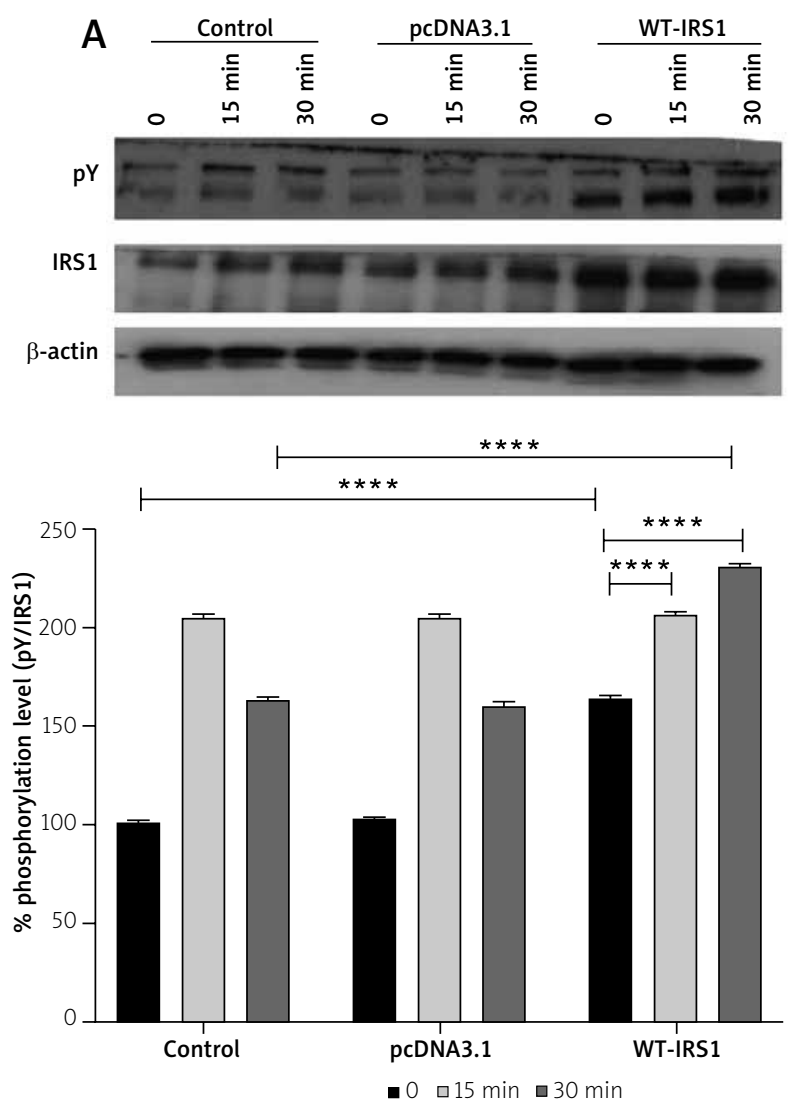

ses, we observed a 20\% increase in Grb2 expression after insulin treatment for $15 \mathrm{~min}$ and $30 \mathrm{~min}$ in control and mock transfected groups compared to their untreated groups however we did not detect any significant changes between control and mock transfected groups in Grb2 expressions (Fig. 1). In the IRS1 overexpressed group, Grb2 expression gradually increased depending on the incubation time of insulin treatment. Expression levels of Grb2 were $40 \%$ and $70 \%$ increased compared to its untreated control after $15 \mathrm{~min}$ and 30 min treatment of insulin, respectively. Although, Grb2 expression elevated by insulin for 15 and $30 \mathrm{~min}$ in control and mock groups, expression levels of Grb2 were higher in ectopic IRS1 overexpressed U-87 MG cells after 15 and 30 min insulin treatment (Fig. 1).

Phosphorylation levels of ERK $1 / 2$ is $10 \%$ increased after treatment of insulin for 15 min compared to the untreated group in control cells (Fig. 2). Its activation level was still the same for 30 min insulin treatment. Although ERK1/2 activation was increased by insu-
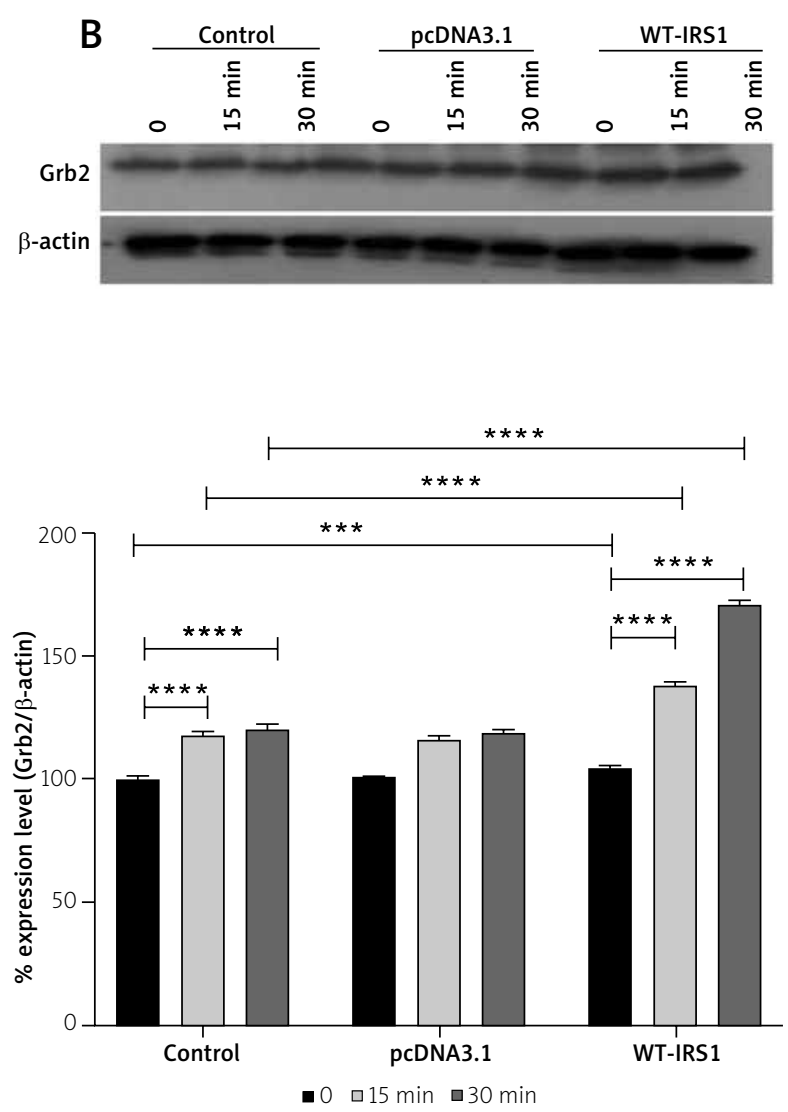

Fig. 1. A) IRS1 tyrosine phosphorylation and B) Grb2 expression levels of U-87 MG cells. (Control: untransfected cells; pcDNA3.1: mock transfected; WT-IRS1: pcDNA3.1-IRS1 transfected; ${ }^{* * *} p<0.005$; $\left.{ }^{* * * *} p<0.0001\right)$. 

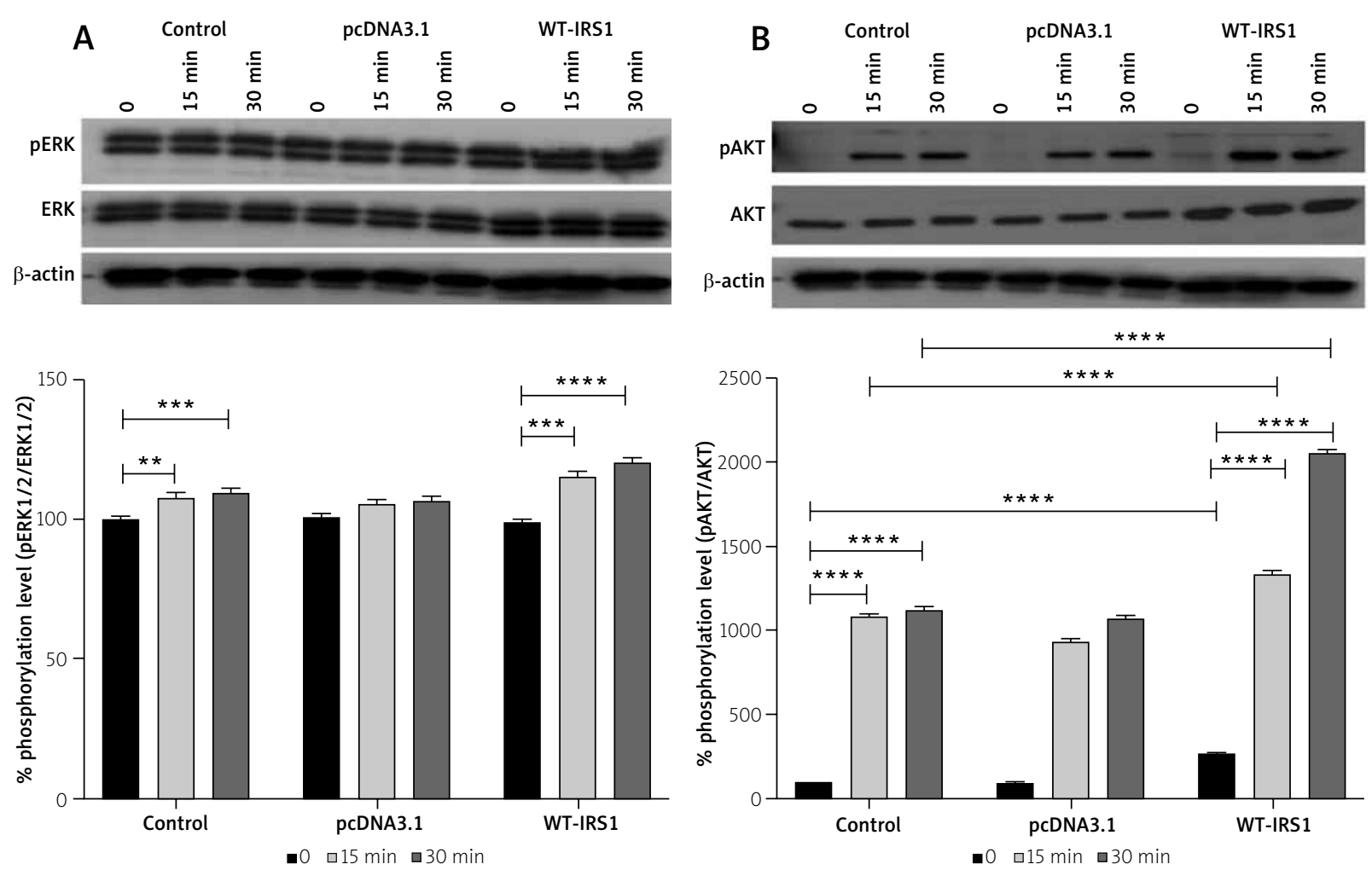

Fig. 2. A) ERK1/2 phosphorylation and B) AKT1 phosphorylation levels of U-87 MG cells. (Control: untransfected cells; pcDNA3.1: mock transfected; WT-IRS1: pcDNA3.1-IRS1 transfected; ${ }^{\star *} p<0.01 ;{ }^{* * *} p<0.005$; $\left.{ }^{* * * *} p<0.0001\right)$.

lin in IRS1 overexpressed U-87 MG cells, we did not observe any significant changes in ERK $1 / 2$ phosphorylation compared to control and mock transfected groups (Fig. 2).

After 15 and 30 min insulin treatment, we detected approximately a 12 -fold increase in AKT1 phosphorylation in control and mock transfected groups (Fig. 2). Interestingly, AKT1 phosphorylation level was 2-fold higher in untreated IRS1 overexpressed cells compared to untreated control and mock transfected cells. We also observed that AKT1 phosphorylation gradually increased depending on the incubation time unlike the levels of AKT1 phosphorylation in control and mock groups (Fig. 2).

\section{Effect of IRS1 overexpression on the viability of U-87 MG cells}

After determining the effects of IRS1 overexpression on insulin signalling, we wanted to determine whether increased IRS1 and AKT1 activations directly affect the viability of U-87 MG cells. Based on the MTT analysis, at the end of $24 \mathrm{~h}$ incubation,

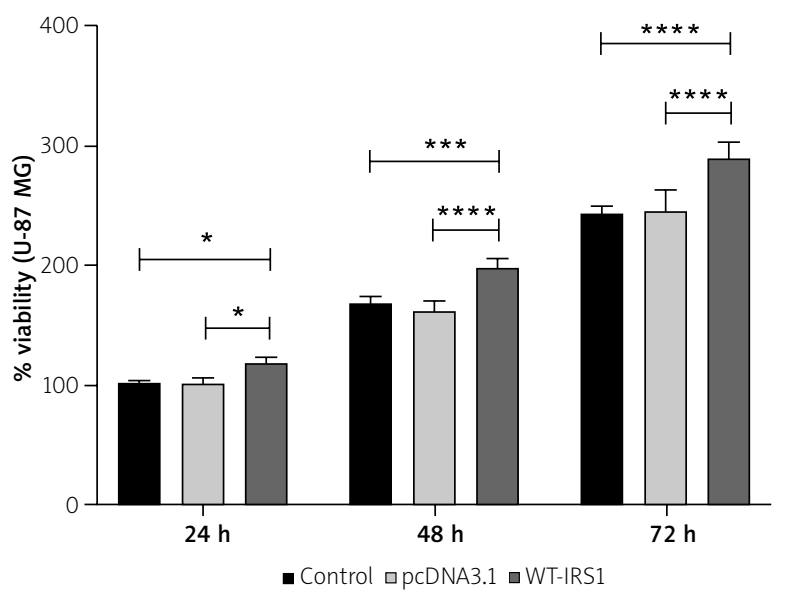

Fig. 3. Viability levels of U-87 MG cells. (Control: untransfected cells; pcDNA3.1: mock transfected; WT-IRS1: pcDNA3.1-IRS1 transfected; ${ }^{*} p<0.05$; $\left.{ }^{* * *} p<0.001 ;{ }^{* * *} p<0.0001\right)$.

viability of U87-MG cells was increased by $18 \%$ in IRS1 transfected cells compared to the control group (Fig. 3). This elevation was gradually increased in the time-dependent manner. $48 \%$ increases were 
detected in IRS1 overexpressed U-87 MG cells compared to the control group at the end of $72 \mathrm{~h}$ (Fig. 3).

\section{Discussion}

Insulin and insulin like growth factor receptors show their mitogenic and metabolic effects on cells through IRS1 protein. IRS1 is the main adaptor molecule in insulin and insulin like growth factor receptor signalling. Although it has no intrinsic kinase activity, it is phosphorylated by receptors it interacts with. Tyrosine phosphorylation of IRS1 induces the activation of two main signalling pathways AKT and ERK which are highly associated with cell proliferation and cell invasion $[25,27]$.

Glioblastoma multiforme is the most common and aggressive type of primary brain tumour. GBM patients with metabolic disorders such as type 2 diabetes have poor prognosis compared to other patients $[5,20]$. Dysregulation of IRS1 signalling is one of the main causes of type 2 diabetes and IRS1 overexpression is strongly associated with cell proliferation [25]. Although the increased expression level of IRS1 and its effect on cell proliferation have been shown by many studies in different cancer types [18], studies that have shown the association between the tyrosine phosphorylation level of IRS1 and cell proliferation are limited. IRS1 activation was significantly increased upon insulin treatment and this activation is important for the metabolic and mitogenic effects of insulin [25]. Based on our findings, ectopic IRS1 overexpression significantly increased the tyrosine phosphorylation level of IRS1 depending on the time of exposure and it has an upregulatory effect on IRS1 tyrosine phosphorylation. In accordance with our findings, ectopic IRS1 expression in MDA-MB-231 led to an increase in tyrosine phosphorylation of IRS1 after estradiol treatment for $96 \mathrm{~h}$ compared to untransfected control cells [19]. Interestingly, we observed a $62 \%$ increase in the tyrosine phosphorylation of IRS1 without insulin treatment in IRS1 overexpressed U-87 MG cells. IRS1 can interact with many receptor tyrosine kinases such as epidermal growth factor receptor (EGFR) through its phosphotyrosine binding domain [7]. Aberrant activation and increased gene copy number of EGFR are the most common genetic changes in GBM. Knowlden et al. showed that increased tyrosine phosphorylation of IRS1 through the interaction with EGFR led to the increase in cell proliferation and has an important effect on the responsiveness of gefitinib therapy in breast cancer cell line [15]. In accordance with this study, increased IRS1 tyrosine phosphorylation without insulin treatment may occur due to the aberrant EGFR signalling in our study. Another cellular mechanism that may affect the activation level of IRS1 without insulin treatment is the production of insulin and IGF ligands in glioblastoma cells $[2,10,12]$. Autocrine effect of these ligands may induce the elevation of IRS1 activation in IRS1 overexpressed cells in the absence of insulin. Therefore, increased IRS1 expression may have a crucial role in the activation of proliferative pathways in GBM cells without any ligands.

After determining the level of IRS1 activation, we wanted to determine whether the activation of IRS1 would directly affect the expression and activation of its downstream signalling elements in U-87 MG cells. Similar to IRS1 activation, Grb2 expression gradually increased depending on insulin exposure time. During insulin signalling, Grb2 binds pYVNI motif of IRS1 that is located in C-terminal of IRS1. This interaction plays a crucial role in RAS and AKT signalling [26]. Therefore, increased IRS1 activation and expression may induce the elevation of Grb2 expression directly.

Increased Grb2 expression triggers the activation of ERK directly and the phosphorylation of AKT via RAS protein [21]. Based on our results, we did not find any changes in ERK $1 / 2$ phosphorylation in GBM cells compared to mock groups. In that case, we thought ectopic IRS1 may induce AKT1 signalling selectively. Therefore, we studied S473 phosphorylation level of AKT1 and it showed similar activation patterns of IRS1 and Grb2. We observed AKT1 activation was significantly increased in IRS1 overexpressed U-87 MG cells without any treatment and its activation gradually increased in the presence of insulin in a time dependent manner. In concordance with our findings, Gong et al. reported that insulin treatment led to an increase in AKT activation in patient derived glioblastoma xenograft lines however they also did not find any changes in ERK $1 / 2$ activation in these samples [12].

Since overexpression of IRS1 induced the activation of AKT1, we wanted to see whether this activation affects cell survival. As shown in Figure 3, IRS1 overexpression significantly increased the viability of U-87 MG cells. Previous studies have shown the role of IRS1 in cell proliferation and viability $[9,14,17]$. 
In addition to these studies, Sakurai et al. showed that inhibiting the tyrosine phosphorylation of IRS1 significantly decreased diethynitrosamine-induced hepatocellular carcinoma cell proliferation through the inhibition of AKT activation [24]. Another study also suggested that insulin-induced AKT activation promotes the cell survival in primary glioblastoma cells [12].

Taken together, our findings suggested that ectopic IRS1 overexpression may induce the cell viability via AKT1 activation in U-87 MG cells.

\section{Acknowledgements}

This study was supported by a grant from Van Yuzuncu Yil University, The Scientific Research Projects Coordination Unit (Project No: TYL 2018-7427), Van, Turkey.

\section{Disclosure}

The authors report no conflict of interest.

\section{References}

1. Chambless LB, Parker SL, Hassam-Malani L, McGirt MJ, Thompson RC. Type 2 diabetes mellitus and obesity are independent risk factors for poor outcome in patients with high-grade glioma. J Neurooncol 2012; 106: 383-389.

2. Clarke DW, Mudd L, Boyd FT, Jr., Fields M, Raizada MK. Insulin is released from rat brain neuronal cells in culture. J Neurochem 1986; 47: 831-836.

3. Copps KD, White MF. Regulation of insulin sensitivity by serine/ threonine phosphorylation of insulin receptor substrate proteins IRS1 and IRS2. Diabetologia 2012; 55: 2565-2582.

4. Dearth RK, Cui X, Kim HJ, Kuiatse I, Lawrence NA, Zhang X, Divisova J, Britton OL, Mohsin S, Allred DC, Hadsell DL, Lee AV. Mammary tumorigenesis and metastasis caused by overexpression of insulin receptor substrate 1 (IRS-1) or IRS-2. Mol Cell Biol 2006; 26: 9302-9314.

5. Derr RL, Ye X, Islas MU, Desideri S, Saudek CD, Grossman SA. Association between hyperglycemia and survival in patients with newly diagnosed glioblastoma. J Clin Oncol 2009; 27: 1082-1086.

6. Dimitriadis G, Mitrou P, Lambadiari V, Maratou E, Raptis SA. Insulin effects in muscle and adipose tissue. Diabetes Res Clin Pract 2011; 93 Suppl 1: S52-59.

7. Fujioka T, Kim JH, Adachi H, Saito K, Tsujimoto M, Yokoyama S, $\mathrm{Ui}$ M. Further evidence for the involvement of insulin receptor substrates in epidermal growth factor-induced activation of phosphatidylinositol 3-kinase. Eur J Biochem 2001; 268: 4158 4168.

8. Gallo M, Muscogiuri G, Felicetti F, Faggiano A, Trimarchi F, Arvat E, Vigneri R, Colao A. Adverse glycaemic effects of cancer therapy: indications for a rational approach to cancer patients with diabetes. Metabolism 2018; 78: 141-154.

9. Garofalo C, Capristo M, Mancarella C, Reunevi H, Picci P, Scotlandi K. Preclinical effectiveness of selective inhibitor of IRS-1/2 NT157 in osteosarcoma cell lines. Front Endocrinol (Lausanne) 2015; 6: 674 .

10. Gerozissis K. Brain insulin: regulation, mechanisms of action and functions. Cell Mol Neurobiol 2003; 23: 1-25.

11. Gibson SL, Ma Z, Shaw LM. Divergent roles for IRS- 1 and IRS- 2 in breast cancer metastasis. Cell Cycle 2007; 6: 631-637.

12. Gong $Y$, Ma Y, Sinyuk M, Loganathan S, Thompson RC, Sarkaria JN, Chen W, Lathia JD, Mobley BC, Clark SW, Wang J. Insulin-mediated signaling promotes proliferation and survival of glioblastoma through Akt activation. Neuro Oncol 2016; 18: 48-57.

13. Gorgisen G, Gulacar IM, Ozes ON. The role of insulin receptor substrate (IRS) proteins in oncogenic transformation. Cell Mol Biol (Noisy-le-grand) 2017; 63: 1-5.

14. Gorgisen G, Hapil FZ, Yilmaz O, Cetin Z, Pehlivanoglu S, Ozbudak $1 \mathrm{H}$, Erdogan A, Ozes ON. Identification of novel mutations of Insulin Receptor Substrate 1 (IRS1) in tumor samples of nonsmall cell lung cancer (NSCLC): Implications for aberrant insulin signaling in development of cancer. Genet Mol Biol 2019; 42: 15-25.

15. Knowlden JM, Jones HE, Barrow D, Gee JM, Nicholson RI, Hutcheson IR. Insulin receptor substrate-1 involvement in epidermal growth factor receptor and insulin-like growth factor receptor signalling: implication for Gefitinib ('Iressa') response and resistance. Breast Cancer Res Treat 2008; 111: 79-91.

16. Lin YC, Hou SC, Hung CM, Lin JN, Chen WC, Ho CT, Kuo SC, Way TD. Inhibition of the insulin-like growth factor 1 receptor by CHM-1 blocks proliferation of glioblastoma multiforme cells. Chem Biol Interact 2015; 231: 119-126.

17. Machado-Neto JA, Fenerich BA, Rodrigues Alves APN, Fernandes JC, Scopim-Ribeiro R, Coelho-Silva JL, Traina F. Insulin Substrate Receptor (IRS) proteins in normal and malignant hematopoiesis. Clinics (Sao Paulo) 2018; 73 (suppl 1): e566s.

18. Mardilovich K, Pankratz SL, Shaw LM. Expression and function of the insulin receptor substrate proteins in cancer. Cell Commun Signal 2009; 7: 14.

19. Mauro L, Salerno M, Panno ML, Bellizzi D, Sisci D, Miglietta A, Surmacz E, Ando S. Estradiol increases IRS-1 gene expression and insulin signaling in breast cancer cells. Biochem Biophys Res Commun 2001; 288: 685-689.

20. Mayer A, Vaupel P, Struss HG, Giese A, Stockinger M, Schmidberger $\mathrm{H}$. Strong adverse prognostic impact of hyperglycemic episodes during adjuvant chemoradiotherapy of glioblastoma multiforme. Strahlenther Onkol 2014; 190: 933-938.

21. Mendoza MC, Er EE, Blenis J. The Ras-ERK and PI3K-mTOR pathways: cross-talk and compensation. Trends Biochem Sci 2011; 36: 320-328.

22. Patil SS, Gokulnath P, Bashir M, Shwetha SD, Jaiswal J, Shastry AH, Arimappamagan A, Santosh V, Kondaiah P. Insulin-like growth factor binding protein-2 regulates beta-catenin signaling pathway in glioma cells and contributes to poor patient prognosis. Neuro Oncol 2016; 18: 1487-1497. 
23. Pollak M. Insulin and insulin-like growth factor signalling in neoplasia. Nat Rev Cancer 2008; 8: 915-928.

24. Sakurai Y, Kubota N, Takamoto I, Obata A, Iwamoto M, Hayashi T, Aihara M, Kubota T, Nishihara H, Kadowaki T. Role of insulin receptor substrates in the progression of hepatocellular carcinoma. Sci Rep 2017; 7: 5387.

25. Shaw LM. The insulin receptor substrate (IRS) proteins: at the intersection of metabolism and cancer. Cell Cycle 2011; 10: 1750-1756.

26. Skolnik EY, Lee CH, Batzer A, Vicentini LM, Zhou M, Daly R, Myers MJ, Jr., Backer JM, Ullrich A, White MF, Schlessinger J. The $\mathrm{SH} 2 / \mathrm{SH} 3$ domain-containing protein GRB2 interacts with tyrosine-phosphorylated IRS1 and Shc: implications for insulin control of ras signalling. EMBO J 1993; 12: 1929-1936.

27. Taniguchi CM, Emanuelli B, Kahn CR. Critical nodes in signalling pathways: insights into insulin action. Nat Rev Mol Cell Bio 2006; 7: 85-96 